\title{
Open APIs for the Rest of Us
}

\author{
Simon Mayer \\ Pro2Future $\mathrm{GmbH}$ and \\ Graz University of Technology \\ Graz, Austria \\ simon.mayer@pro2future.at
}

\begin{abstract}
The creation of open APIs is one of the core concepts advocated by the Web of Things community. However, drastically open APIs to the basic functional components of physical devices are only found in research projects and seem to not yet find favor with a wider audience. With this paper, I would like to initiate a discussion about the value of open APIs for the producers and users of smart devices, and for society as a whole: How can manufacturers be motivated to outfit their products with open APIs? How can consumers be educated about the benefits of not being locked into manufacturer ecosystems? And might the decision of opening up a device API even be subject to antitrust law?
\end{abstract}

\section{Author Keywords}

Web of Things, Internet of Things, Open APIs

\section{INTRODUCTION}

Over the past decade, we have witnessed the rapid proliferation of concepts at the core of the Web of Things (WoT) in the research community. In research projects in the Internet of Things domain, it is now commonplace that physical devices and their components are equipped with open Web APIs and, routinely, their behavior is implemented using scripting languages on remote servers. Sometimes, this basic setup is extended with more features that are recommended by researchers in the WoT domain, for instance with thought-out REST APIs to the thing's properties, actions, and events to simplify their usage by human and machine clients ${ }^{1}$, semantic markup to facilitate their (automatic) composition [6], current security features ${ }^{2}$ and measures for ensuring the compatibility of applications with resource-constrained environments [3].

In this position paper, I focus on the basic enabler for all of these features: things that provide open Web APIs to their individual components. In its purest form, this concept has

\footnotetext{
${ }^{1}$ More information is available at http://w3c.github.io/wot/ current-practices/wot-practices.html

${ }^{2}$ A current overview of security and privacy mechanisms for the WoT is available at https://Www.w3.org/WoT/IG/wiki/Design-Time_ Security\%26Privacy_Means
}

Permission to make digital or hard copies of part or all of this work for personal or classroom use is granted without fee provided that copies are not made or distributed for profit or commercial advantage and that copies bear this notice and the full citation on the first page. Copyrights for third-party components of this work must be honored. For all other uses, contact the owner/author(s).

WoT 2017 October 22, 2017, Linz, Austria

(C) 2017 Copyright held by the owner/author(s).

ACM ISBN 123-4-5678.

DOI:

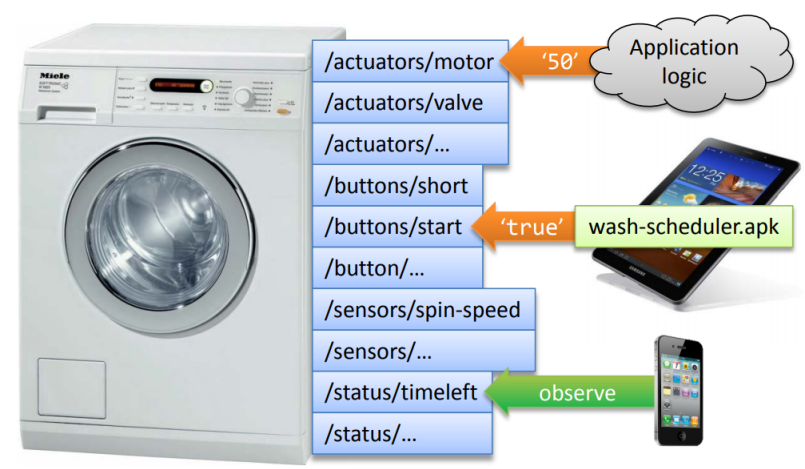

Figure 1. A washing machine whose every button, actuator, and sensor is modeled as a Web resource (image from [4]).

been discussed in the 2012 paper Moving Application Logic from the Firmware to the Cloud: Towards the Thin Server Architecture for the Internet of Things that proposed to utilize thing-embedded servers merely as wrappers for device sensors and actuators - the application logic is provided by (remote) application servers that leverage the open APIs provided by the devices in question, potentially in conjunction with other Web resources [4]. In that paper, we suggested as an example a washing machine that provides Web APIs to every button, actuator, and sensor but does not store or run any washing program locally (see Fig. 1). Rather, the resources of the appliance interact with a service that hosts the decision logic. Benefits of this approach include continuous optimization of the washing programs and over-the-air introduction of new features (e.g., to optimize resource consumption), facilitated mashing up with other devices and services (e.g., for sending notifications), and continuous monitoring of the machine's behavior (e.g., for predictive maintenance). These do not only benefit the end consumer: washing machine suppliers are suddenly put into a position that allows them to sell washing-as-a-service and thereby escape the "box-selling" business where margins might be squeezed by competitors. In addition, the washing machine can, in this way, easily be used as a point-of-sale (for detergent, for instance) and profiling of consumer behavior might help to adapt the products to local market demand and to optimize the supply chain. Finally, suppliers (and users) might profit from their products participating in regulatory or market-driven incentive schemes, such as demand-side energy management. On a societal scale, the benefits of open APIs to such thin servers are obvious: heavy competition for providing the best application logic would heavily benefit consumers by depressing prices and boosting customer value. 
To unlock these benefits, products and their components need to feature APIs that are not only open to their manufacturers' own back ends but can be programmed against by anyone, ideally even by users (perhaps supported by end-user programming tools). Opening up APIs in this way has started to become mainstream for many digital services - recently, experts have even expressed the opinion that providers might have an obligation to open up APIs, subject to antitrust law ${ }^{3}$. While the technical feasibility of the proposed concepts is clear, with this paper, I would like to start a discussion about how we researchers and standardization experts might be able to bring open APIs to industry and consumer products, ideally to the extent described in [4]. In the rest of this paper, I attempt to summarize the main benefits of open APIs for consumers, producers, and society - specifically, I focus on the home automation, industrial automation, and building automation domains. Together with technological and regulatory solutions to the main drawbacks that open APIs entail (e.g., for manufacturers, weakening customer lock-in to a physical product), this should help to distill the main arguments for convincing stakeholders to adopt open APIs in practice.

\section{WHY OPEN APIS: CONVINCING STAKEHOLDERS}

The stakeholders who need to be convinced before open APIs to physical devices can become mainstream are the suppliers/producers and buyers/consumers of electronic devices in the aforementioned three domains. Ideally, we could show that outfitting a device with an open API by itself holds enough value for the device's manufacturer (e.g., by supporting its digitalization agenda or enabling monitoring of the device and its optimization; see Section Open APIs for Disruptive Manufacturers) while at the same time benefiting its customers enough for commanding a premium over a similar device without that feature (see Section Open APIs for Knowledgeable Consumers). We also need to consider the societal perspective (see Section Open APIs as a Societal Opportunity): deterring manufacturers from locking their customers into their own ecosystems is desirable as it should increase the overall value of the products in question since the increased competition for delivering digital-physical services should lead to more valuable offerings at lower prices for customers.

\section{Open APIs for Disruptive Manufacturers}

For many manufacturers of formerly purely physical products, open APIs form a cornerstone in the transformation of their business from "selling boxes" to "selling services" (which ideally should entail higher margins) - as discussed in the Introduction in the context of a fully open home appliance, opening up device APIs will not only enable device makers to deliver a wide range of IT-based services on top of the basic Thing-based function and open up new business models that used to be exclusive to digital services [2], but in addition allow them to monitor, manage, and optimize their products, sometimes summarized as High-Resolution Management [8].

However, in many domains, opening up devices in this way is unthinkable at this point, as manufacturers would lose their

\footnotetext{
${ }^{3}$ In this case concerning the UBER service restricting access for price comparison services: http://www . benedelman . org/news/053116-1. html
}

ability to lock customers into their ecosystems - in our living rooms, the battle to get a Smart Home Gateway into users' homes (using TVs, WiFi routers, thermostats and, more recently, loudspeakers as beachheads) has been going on for more than a decade, with limited success. The same basic principle also holds for economically more relevant domains such as the management of commercial buildings and, importantly, industrial automation scenarios: here, it would however be fascinating (and very valuable) to have production equipment implement open APIs to their full extent as this would enable machines to compose their abilities on the fly and thereby enable factories to produce mass-customized products at massproduction prices [5]. If researchers and developers could find ways to overcome the challenges that remain for such flexible compositions to happen at runtime, the economic benefits should outweigh the ability to create closed ecosystems, thus making open APIs an economically viable alternative. Fortunately, steps in that direction are already being taken by several industrial associations, for instance the German Electrical and Electronic Manufacturers' Association (ZVEI) with its proposal of an Asset Administration Shell [7].

Regarding building automation scenarios, it seems that the traditional players Honeywell, Johnson Controls, etc. are currently being coerced by new market entrants to open up their devices and provide innovative digital services on top of them - also in this domain, services such as IFTTT ${ }^{4}$ have shown that manufacturers seem to be willing to open up their devices in principle.

\section{Open APIs for Knowledgeable Consumers}

From the perspective of consumers across domains, products that feature open APIs should in principle be functionally and economically favorable: they give them the possibility to have service providers compete with each other about who can provide the most valuable digital-physical service, which should lead to increasing consumer value and decreasing prices. If these benefits could be efficiently communicated (perhaps using an "Open API" or "WoT Ready" certification program), it is conceivable that consumers would pay a premium for devices that feature open APIs and thereby do not lock them into a specific ecosystem. An additional benefit for customers in the home automation domain is the provisioning of - and paying for-devices as-a-service. The same logic holds true in the industrial and building automation domains: from a consumer perspective, the main obstacle to motivating manufacturers to open up their devices discussed in Section Open APIs for Disruptive Manufacturers turns into its main benefit as consumers profit heavily from interoperable products.

The main obstacle to increased customer demand for open products most probably is neither functional nor (directly) economic, but is rooted in privacy and security concerns: customers need to trust products that are labeled "WoT Ready." While the technological means for sufficiently securing products with open APIs are available, it is necessary that manufacturers implement current security features in their products. In addition, we require a regulatory framework that governs

\footnotetext{
${ }^{4}$ See https://ifttt.com/
} 
ownership of the generated data. However, both these challenges are already manifest when outfitting devices with basic connectivity features and are thus not an obstacle to open APIs per se.

\section{Open APIs as a Societal Opportunity}

On a societal level, I believe that a proliferation of open APIs would be beneficial in a plethora of ways that range from fostering innovative uses of existing hardware to higher utilization of products and thus reduced resource usage. It might even be the case that the issue of API Openness forms part of antitrust jurisdiction: similar to blocking providers of operating systems to pre-install their own browsers and banning search services from unfairly steering customers to their favored shopping platforms, manufacturers might in the future be mandated to deliver their products without digital or physical lock-in features. Recent controversy about UBER's practice of restricting API access for price comparison services has some experts arguing that companies that use UBER's API act as de-facto distributors - thus framing the company as a dominant firm that imposes exclusivity requirements along its distribution channel: this ultimately harms the customer as exclusive-dealing contracts may slow a potential rival's expansion by requiring it to develop alternative outlets for its product or rely on inferior or more expensive outlets, thus delaying the rival's growth and harming innovation [1]. As pointed out by one of the reviewers of this paper, a somewhat less aggressive (but still controversial) approach to coercing producers into providing open APIs would be to create hardware/software licenses that impose open APIs, in a way similar to how copyleft works in the open source software domain.

\section{CONCLUSION}

Products that feature drastically open APIs are beneficial for consumers and society as a whole, and also carry a number of advantages for their manufacturers. However, makers and buyers need to be aware of and convinced about these benefits and several technical and regulatory challenges need to be overcome to motivate both, consumers and producers, to create and use these products. With this paper, I would like to start a discussion about how this can be accomplished.

\section{REFERENCES}

1. Phillip E. Areeda and Herbert Hovenkamp. 2011. Fundamentals of Antitrust Law. Aspen Publishers, New York, NY, USA.

2. Elgar Fleisch, Markus Weinberger, and Felix Wortmann. 2014. Business Models and the Internet of Things. In Proc. Int. Workshop on Interoperability and Open-Source Solutions for the Internet of Things. 6-10.

3. Matthias Kovatsch. 2013. CoAP for the Web of Things: From Tiny Resource-constrained Devices to the Web Browser. In Proc. UbiComp (Adjunct). ACM, New York, NY, USA, 1495-1504.

4. Matthias Kovatsch, Simon Mayer, and Benedikt Ostermaier. 2012. Moving Application Logic from the Firmware to the Cloud: Towards the Thin Server Architecture for the Internet of Things. In Proc. IMIS. 751-756.

5. Simon Mayer, Dominic Plangger, Florian Michahelles, and Simon Rothfuss. 2016a. UberManufacturing: A Goal-Driven Collaborative Industrial Manufacturing Marketplace. In Proc. IoT. 111 - 119.

6. Simon Mayer, Ruben Verborgh, Matthias Kovatsch, and Friedemann Mattern. 2016b. Smart Configuration of Smart Environments. IEEE Trans. Autom. Sci. Eng. 13, 3 (2016), 1247-1255.

7. Plattform Industrie 4.0. 2016. Structure of the Administration Shell. Federal Ministry for Economic Affairs and Energy, Berlin, DE.

8. Brian Subirana, Sanjay Sarma, and Elgar Fleisch. 2006. High-Resolution Management: Improving Management Vision. IESE Alumni Magazine 102 (2006), 8 - 13. 